\title{
Reflets
}

Revue ontaroise d'intervention sociale et communautaire

\section{La promotion de la santé : des pas de géants au-delà de l'an 2000}

\section{Hélène Gagné}

Volume 5, numéro 2, automne 1999

La santé des francophones de l’Ontario

URI : https://id.erudit.org/iderudit/026277ar

DOI : https://doi.org/10.7202/026277ar

Aller au sommaire du numéro

Éditeur(s)

Reflets : Revue ontaroise d'intervention sociale et communautaire

ISSN

1203-4576 (imprimé)

1712-8498 (numérique)

Découvrir la revue

Citer cet article

Gagné, H. (1999). La promotion de la santé : des pas de géants au-delà de l'an 2000. Reflets, 5(2), 192-201. https://doi.org/10.7202/026277ar

Tous droits réservés (C) Reflets : Revue ontaroise d'intervention sociale et communautaire, 1999
Ce document est protégé par la loi sur le droit d'auteur. L'utilisation des services d'Érudit (y compris la reproduction) est assujettie à sa politique d'utilisation que vous pouvez consulter en ligne.

https://apropos.erudit.org/fr/usagers/politique-dutilisation/ 


\section{La promotion de la santé : des pas de géants au-delà de l'an 2000}

\section{$H$ élène $\mathbf{G}$ agné}

C onsultante en promotion de la santé pour le $\mathrm{C}$ entre ontarien d'information en prévention (C O IP)

À l'aube du nouveau millénaire, les espoirs, parfois même prophétiques, sont à leur apogée. Plusieurs spéculent sur les changements à venir en ce qui concerne la présence accrue de la technologie dans nos vies quotidiennes, les effets de la mondialisation de l'économie et de la compétition, les courants politiques et leurs répercussions mondiales et enfin, l'impact de tous ces changements sur le mode de vie et la santé des individus. Puisque l'avènement du nouveau millénaire pousse à la réflexion et à l'introspection professionnelle, je me lance également dans la mêlée en posant un regard global sur la promotion de la santé et sur ce que l'avenir réserve à ce domaine.

Le présent article explore donc lestendances passées, présentes et futures en promotion de la santé d'après les observations et l'expertise de quelques intervenants impliqués dans le domaine. $\mathrm{N}$ ous avons recueilli leurs commentaires lors d'entrevues.

\section{Se rafraîchir la mémoire}

$D$ 'après I rving $\mathrm{R}$ ootman, directeur général du $\mathrm{C}$ entre for $\mathrm{H}$ ealth Promotion de l'U niversité deToronto, l'idée de promotion de la santé n'a pas plus de vingt-cinq ans. Elle remonte à la publication du rapport Lalonde sur la santé. Dans ce document, l'auteur propose que l'amélioration de l'état de santé des Canadiens dépasse le seul système de distribution des soins et inclut aussi des facteurs liés au milieu dans lequel les individus vivent. À 
l'époque, on mettait surtout l'accent sur les habitudes de vie saine dans l'espoir que les gens puissent assumer leurs responsabilités et changer leurs mauvaises habitudes. A insi, le tour serait joué. La population s'en porterait mieux et les coûts associés aux soins médicaux diminueraient par surcroît. I rving R ootman mentionne qu'on allait même jusqu'à croire, à l'époque, que si les gens connaissaient les conséquences néfastes sur la santé de leurs mauvaises habitudes, ils cesseraient définitivement ces comportements malsains à la faveur de comportements plus sains.

Acceptant cette prémisse, le gouvernement fédéral s'est ensuite lancé dans une cabale publicitaire sur la consommation modérée d'alcool, sur les bienfaits de l'activité physique ou sur les dangers du tabagisme, etc. C ette publicité visait à diffuser les renseignements nécessai res sur les modes de vie saine pour que les gens puissent prendre des décisions informées. $M$ al heureusement, la campagne publicitaire du gouvernement fédéral n'a pas eu les résultats escomptés. Comme nous le savons bien aujourd'hui, il est important de sensibiliser et d'informer la population sur des questions de promotion de la santé et de prévention, mais cette stratégie ne suffit pas à produire des changements de comportement aux niveaux individuel et collectif.

C'est de ce constat, à savoir que certains facteurs échappant au contrôle décisionnel de l'individu influencent également la santé, qu'est né l'intérêt pour une approche plus holistique, comme le mentionne I rving $R$ ootman dans notre entretien. C e sont aussi de telles réflexions qui ont donc conduit au développement de la C harte d'O ttawa (1986) qui prône une démarche à différents niveaux : niveau environnemental, niveau politique, niveau de santé publique, niveau communautaire et niveau individuel. La C harte d'0 ttawa définit la promotion de la santé comme un processus qui confère aux populations les moyens d'assurer un plus grand contrôle sur leur propre santé, et d'améliorer celle-ci. Lesstratégies suggérées dans la $\mathrm{C}$ harte afin de promouvoir la santé de tous, comprennent l'élaboration de politiques publiques saines, la création de milieux favorables, le renforcement de l'action 
communautaire, le développement de compétences et la réorientation des services de santé. La $\mathrm{C}$ harte d'O ttawa met ainsi l'accent sur l'environnement dans lequel les individus vivent et travaillent plutôt que de tenter d'influencer le comportement individuel de manière isolée.

Irving R ootman précise que la première initiative mettant ces concepts théoriques en pratique fût le mouvement des communautés en santé. C e mouvement d'origine internationale a été particulièrement populaire au Q uébec, suivi de la C olombieB ritannique et de I'O ntario. Comme son nom l'indique, les communautés en santé sont un réseau de communautés et d'organismes qui partagent un même but, celui de créer des communautés en santé. Les stratégies principales utilisées pour créer des communautés en santé sont le développement communautaire et l'action locale. Le tout devait favoriser en bout de ligne la prise en charge communautaire. Le gouvernement fédéral et les gouvernements provinciaux de l'heure ont appuyé cette initiative dès le départ et leurs succès ont été reconnus internationalement. $D$ epuis lors, le gouvernement fédéral a retiré son appui tandis que celui du gouvernement de l'O ntario a considérablement faibli.

\section{La tendance à la vogue}

Q uelle serait la nouvelle tendance à la mode? D epuis les cinq dernières années, nous entendons parler du concept de «santé de la population ». Celui-ci a pratiquement éclipsé le concept de promotion de la santé et ce, particulièrement au niveau gouvernemental, comme le souligne Irving R ootman. C elui-ci soutient que le concept de la santé de la population semble davantage prendre racine pour deux raisons principales: 1 ) nous avons échoué dans nos efforts pour démontrer, preuve à l'appui, aux fonctionnaires que la promotion de la santé fonctionne bel et bien et que c'est un investissement sûr et, 2) les arguments avancés par des scientifiques et des économistes, ayant une crédibilité plus grande que lesgens impliquésen promotion de la santé, ont réussi à convaincre les fonctionnaires que la santé de la population s'améliorerait davantage en travaillant dans les secteurs 
autres que le secteur traditionnel de la santé. Q ui plus est, la collaboration intersectorielle, selon leurs dires, serait la stratégie idéale pour mettre en pratique le concept de la santé de la population.

Toutefois, la collaboration intersectorielle est beaucoup plus difficile à accomplir en pratique qu'en théorie, ajoute Irving $\mathrm{R}$ ootman. $\mathrm{O} r$, ironie du sort, les histoires à succès sur l'action intersectorielle se déroulent davantage au niveau communautaire et principalement, lorsque le secteur de la santé a son mot à dire dans la prise de décisions. En fait, si la réussite des actions communautaires peut servir à donner ses lettres de noblesse au mode d'action intersectorielle, au point où celui-ci s'impose dans nos politiques publiques, par un effet de rétroaction, cela facilitera encore plus l'action intersectorielle déjà amorcée au niveau communautaire.

A ux dires de Irving $R$ ootman, la nouvelle vague en promotion de la santé semble accorder de l'importance au fait de mettre I'individu au centre de l'action. 0 r, la promotion de la santé n'at-elle pas toujours prôné l'importance du développement des compétences et la prise en charge personnelle et communautai re? Et, la meilleure façon d'accomplir ces objectifs est toujours le développement communautaire dans sa tradition la plus pure. $\mathrm{Du}$ moins, c'est à une conclusion semblable que parviennent I rving $R$ ootman and John $R$ aeburn dans leur livre intitulé, People $C$ entred $\mathrm{H}$ ealth Promotion.

\section{La préoccupation d'aujourd'hui: preuve à l'appui}

Peggy Schultz, consultante en promotion de la santé au COIP, déclare que la promotion de la santé est davantage une façon de pensée qu'un domaine de travail bien délimité. Elle soutient que la promotion de la santé est également un processus et un résultat. D ansce sens, on devrait se demander si le domaine de la promotion de la santé $n$ 'est pasune transition vers autre chose. La promotion de la santé pourrait tout simplement être une philosophie particulière qui permet de faire desliens entre différents domaines ou secteurs de travail, mentionne Peggy Schultz. 
La plupart des domaines professionnels requièrent des théories, un ensemble de recherches, d'expériences, de structures et de professionnels qui y travaillent. Le combat de la promotion de la santé a été bien longtemps de trouver une théorie générale pour expliquer son travail alors qu'en réalité, il existe plusieursthéories qui guident la pratique. Selon Peggy Schultz, cette situation est source de grande complexité au niveau des stratégies et des approches, ce qui rend difficile la démonstration de la pertinence et la mesure de l'impact des interventions en promotion de la santé. À l'heure actuelle, quelques groupes d'intervenants dans le domaine sont en train de relever le défi en définissant le processus et les résultats de nos meilleures pratiques en promotion de la santé et en développant un cadre de référence pour l'évaluation de nos interventions. C es efforts pour ront sans doute apporter de l'eau au moulin de la promotion de la santé.

De son côté, Alison Stirling, consultante en promotion de la santé au COIP, explique par le désir de pouvoir atteindre des résultats tangibles et mesurables, la tendance actuelle en promotion de la santé de revenir aux habitudes de vie saine. Elle suggère également que le gouvernement préfère l'approche des habitudes de vie saine pour la simple raison que la responsabilité du changement incombe davantage à l'individu et que l'intervention nécessaire pour susciter de tels changementss'applique à un groupe cible ou témoin. D 'une certaine façon, l'approche des habitudes de vie saine préserve le statut quo, présume que nous pouvons mesurer les impacts de nos interventions et cadre bien avec le modèle médical traditionnel.

Bien que la promotion de la santé semble accuser une baisse de popularité, tout particulièrement au niveau gouvernemental, plusieursindices soulignent sa progression. En effet,A lison Stirling soutient que l'intérêt pour les facteurs déterminants de la santé a réussi à ouvrir l'esprit sur la façon de concevoir la santé. D orénavant, la santé n'est plus le résultat ou un état à atteindre et à améliorer, mais plutôt l'ensemble desfacteurs qui influencent la santé, tels que l'environnement, les aspects sociaux, économiques, politiques, etc. En d'autres termes, nous portons davantage 
attention aux facteurs qui composent et maintiennent la santé, histoire d'adopter une perspective plus large.

De son côté, I rving R ootman est confiant que le domaine de la promotion de la santé accomplit beaucoup de bonneschoses àl'heure actuelle. À son avis, nous avons accompli du bon travail en ce qui touche le fait d'avoir de meilleures pratiques en promotion de la santé: évaluation, échange d'information, éducation et formation, développement des compétences individuelles et communautaires et ce, avec ou sans soutien officiel.

\section{Pleins feux sur l'avenir}

II faut admettre que nos accomplissements semblent minces à la lumière de l'ampleur des problèmes dans le monde. Cette réalisation ne doit pas nous absoudre de notre responsabilité et d'essayer de faire de notre mieux, déclare Irving R ootman. En fait, il pense que les gens impliqués dans le domaine de la promotion de la santé peuvent apporter une large contribution dans les secteurs suivants:

1) Grâce aux mouvements comme celui des communautés en santé et aux efforts en promotion de la santé, nous pouvons aider les individus et les communautés à construire une image positive de l'avenir où les gens auront les moyens de surmonter leur sentiment d'impuissance et d'initier des actions positives vers des changements sains.

2) N ous pouvons réaliser ces images positives de l'avenir grâce au développement communautaire en mettant à profit nos connai ssances, nos recherches et nos expériences passées. A près tout, nous avons connu de grands succès avec cette stratégie. La prochaine étape consiste à consolider nos résultats et les diffuser aux gens qui peuvent en faire bon usage.

3) Au fil des ans, les gens impliqués dans le domaine ont acquis une expertise et des connaissances en promotion de la santé. D e plus, nous possédons maintenant une infrastructure propre à favoriser le développement de la compétence de ces individus, tout en ayant à cœur le souci de former la relève. 
4) De plus en plus, un réseau mondial d'individus impliqués en promotion de la santé partage les mêmes val eurs et principes. Ils apprennent à travailler ensemble pour un but commun. Les outils de communication électronique comme Internet et les bulletins électroniques axés sur la promotion de la santé, ont grandement facilité l'échange d'information et ont concouru à ancrer solidement nos valeurs et principes de travail.

5) Il existe également d'autres ressources à notre disposition telles que des sources de financement pour la recherche et le développement de programmes. $\mathrm{N}$ otre capacité à créer des liens entre les différents secteurs d'activités nous a permis de développer une expertise pour trouver des fonds auprès de sources alternatives.

6) Finalement, le travail provenant du domaine de la promotion de la santé offre de l'espoir. $N$ otre habileté à réaliser concrètement cet espoir sera garante de l'essor que connaîtra la promotion de la santé dans les années à venir.

L'espoir de Peggy Schultz est que les actionsmenées en promotion de la santé permettent un jour la création de liens entre les différents secteurs d'activités et, ainsi, contribuer à la mise en place d'une vision plus holistique de la santé pour tous. Par exemple, l'intérêt pour les facteurs déterminants de la santé nous incitera davantage à faire le lien entre l'environnement et la santé. Elle renchérit sur ce point en donnant comme exemple, la création d'un terrain de golf et ses effets environnementaux. Si agréable qu'elle puisse être pour nosloisirs, la création d'un terrain de golf transforme artificiellement le paysage et les produits chimiques employés à cette fin ont un impact sur la santé collective. D onc, tant et aussi longtemps que nous ne ferons pas attention aux liens directs entre nosactions et leurs conséquences, nous éprouverons de la difficulté à travailler de façon multidimensionnelle et responsable.

De son côté, Alison Stirling illustre l'importance d'intégrer plusieurs dimensions de la santé en citant le travail quotidien des intervenants œuvrant en promotion de la santé. Son action auprès des intervenants l'amène à constater leur surcharge de 
travail et l'ampleur des problèmes auxquels ils ont à faire face. II est important de réaliser que les intervenants ne peuvent pas travailler sur tous les fronts en même temps. Les intervenants doivent reconnaître que certaines questions ne relèvent pas de leur travail, voire même de leur responsabilité, nous dit-elle. C ela dit, ils peuvent au moins établir lesliens entre les différentes questions qui influencent la santé. $\mathrm{N}$ e serait-ce que cela, ce serait beaucoup. Et leur contribution pourrait être tout simplement de partager ces histoires avec leurs collègues afin qu'éventuellement d'autres liens puissent se créer entre leur travail et les groupes d'intérêt.

\section{Un regard sur l'Ontario}

D epuis environ deux ans, le gouvernement de l'O ntario cherche à développer un système de promotion de la santé qui permettrait de regrouper les différents centres de ressources. L'idée derrière cette initiative, outre la réduction anticipée de coûts, est d'être en mesure d'avoir une approche plus coordonnée dans nos interventions en promotion de la santé. Pour ce faire, le gouvernement encourage une collaboration plusétroite entre les différents centres de ressources qu'il subventionne, soit environ 14 centres. C eux-ci diffèrent les uns des autres sous plusieurs aspects: infrastructure, stratégies d'intérêt (p. ex. la promotion de la santé en général, le tabagisme, la santé cardiaque, l'activité physique), leur population cible (p. ex. le public, les intervenants, etc.) et leurs services (p. ex. formation, consultation, réseautage, échange d'information, etc.).

Alison Stirling accueille favorablement la possibilité de coordonner les efforts de différents centres de ressources, surtout si ces efforts mènent au développement de stratégies communes en promotion de la santé.Toutefois, elle précise que la promotion de la santé ne doit pas se limiter à ces seuls centres et doit voir plus large encore.Ainsi, les actions en promotion de la santé doivent inclure les intervenants œuvrant dans les centres de santé communautaires, dans le centre de santé publique, dansles secteurs de l'environnement, de l'éducation et bien d'autres. D'ailleurs, aux dires de Peggy Schultz, ces secteurs d'activité abordent déjà 
certaines problématiques portant sur certains déterminants de la santé. II s'agit donc de créer des liens afin d'établir des fondations plus solides entre les divers organismes.

Pour ce faire, le COIP et le $\mathrm{C}$ entre for $\mathrm{H}$ ealth Promotion encourage les échanges d'information et la collaboration entre les organismes dans l'espoir que la promotion de la santé puisse avoir un impact durable et créer les changements nécessaires pour le bien-être de la population. Ces relations et ces échanges, si souhaitables soient-ils pour le travail en promotion de la santé, ne doivent pas occuper le premier rang. En effet, selon Irving $\mathrm{R}$ ootman, nous pouvons accomplir notre travail en promotion de la santé si nous ne perdons pas de vue l'objectif initial qui consiste à améliorer la santé des gens dans leur ensemble. De toute évidence, chaque organisme et chaque intervenant utilisent des approches différentes en matière de promotion de la santé, mais l'important, c'est que tous s'entendent et convergent pour favoriser le mieux-être collectif. Par ailleurs, I rving R ootman nous incite à la vigilance par rapport à notre mission initiale en promotion de la santé afin, le jour venu, de pouvoir maintenir le cap lorsque les courants politiques chercheront à nousfaire dévier de notre parcours.

C ela dit, les tâches qui nous attendent sont celles de créer des alliances au-delà des secteurs traditionnels de la santé et des services sociaux et de préparer également une relève en promotion de la santé afin de poursuivre le travail avec de nouvelles perspectives. II y a donc beaucoup de pain sur la planche en promotion de la santé avec le nouveau millénaire qui s'annonce.

Sur un plan plus personnel, je dois avouer que mes entretiens avec I rving, Peggy et A lison ont réveillé mon enthousiasme pour le travail en promotion de la santé. Je suis toujours étonnée de voir à quel point le travail quotidien peut nous submerger et comment il peut être bénéfique de s'arrêter un bref moment pour discuter avec des collègues dans le domaine. Je souhaite aux lecteurs de pareils moments de découverte pour l'an 2000. 
Reflets $\square-\curvearrowleft$ W. $5, n^{\circ} 2$, automne 1999

Des pratiques à notre image

\section{Bibliographie}

LALO N DE, M arc (1974). N ouvelle perspective de la santé des C anadiens, O ttawa, Gouvernement du Canada.

R AEBUR N, John et Irving RO OTM AN (1988). People-C entred H ealth Promotion, Toronto, John Wiley \& Sons. 